Research Article

\title{
On the Kampé de Fériet Hypergeometric Matrix Function
}

\author{
Ashish Verma $\mathbb{D},{ }^{1}$ Jihad Younis $\mathbb{D},^{2}$ and Hassen Aydi $\mathbb{D}^{3,4,5}$ \\ ${ }^{1}$ Department of Mathematics, Veer Bahadur Singh Purvanchal University, Jaunpur, India \\ ${ }^{2}$ Department of Mathematics, Aden University, Aden, Yemen \\ ${ }^{3}$ Université de Sousse, Institut Supérieur d'Informatique et des Techniques de Communication, H. Sousse 4000, Tunisia \\ ${ }^{4}$ Department of Mathematics and Applied Mathematics, Sefako Makgatho Health Sciences University, \\ Ga-Rankuwa, South Africa \\ ${ }^{5}$ China Medical University Hospital, China Medical University, Taichung 40402, Taiwan
}

Correspondence should be addressed to Hassen Aydi; hassen.aydi@isima.rnu.tn

Received 18 March 2021; Revised 25 March 2021; Accepted 30 July 2021; Published 18 August 2021

Academic Editor: Sergio Ortobelli Lozza

Copyright (C) 2021 Ashish Verma et al. This is an open access article distributed under the Creative Commons Attribution License, which permits unrestricted use, distribution, and reproduction in any medium, provided the original work is properly cited.

In this study, we derive recursion formulas for the Kampé de Fériet hypergeometric matrix function. We also obtain some finite matrix and infinite matrix summation formulas for the Kampé de Fériet hypergeometric matrix function.

\section{Introduction}

The theory of special functions is closely related to the theory of Lie groups and Lie algebras, as well as certain topics in mathematical physics. Symbolic computation and engineering problems usually recognize the majority of special functions. Recently, there has been a surge in the study of recursion formulas for multivariable hypergeometric functions. Recursion formulas for the Appell function $F_{2}$ have been investigated by Opps et al. [1], followed by Wang [2], who presented the recursion relations for all Appell functions. Furthermore, recursion formulas for variant multivariable hypergeometric functions were presented in [3-6]. One can refer to various sources $[7,8]$ for the in-depth study of the hypergeometric functions for several variables.

The theory of generalized matrix special functions has witnessed a rather significant evolution during the last two decades. The reasons of interest have a manifold motivation. Restricting ourselves to the applicative field, we note that for some physical problems, the use of new classes of matrix special functions provided solutions hardly achievable with conventional analytical and numerical means. Special matrix functions appear in the literature related to statistics [9], Lie theory [10], and more recently in connection with the matrix version of Laguerre, Hermite, and Legendre differential equations and the corresponding polynomial families [11-13]. In [14], recursion formulas and matrix summation formulas for Srivastava's triple hypergeometric matrix functions are obtained.

The study is organized in the following manner. In Section 2, we list basic definitions that are needed in the sequel. In Section 3, we obtain recursion formulas for the Kampé de Fériet hypergeometric matrix function (its abbreviation is $\mathrm{K}$ de FHMF). In Section 4, we present finite matrix summation formulas for the (K de FHMF) by applying a derivative operator. Finally, in Section 5, we establish infinite matrix summation formulas for the (K de FHMF).

\section{Preliminaries}

Let $\mathbb{C}^{r \times r}$ be the vector space of $r$ square matrices with complex entries. For any matrix $H \in \mathbb{C}^{r \times r}$, its spectrum $\sigma(H)$ is the set of eigenvalues of $H . H$ in $\mathbb{C}^{r \times r}$ is called a positive stable matrix if $\mathfrak{R}(\lambda)>0$ for all $\lambda \in \sigma(H)$.

The reciprocal gamma function $\Gamma^{-1}(\theta)=1 / \Gamma(\theta)$ is an entire function of the complex variable $\theta$. The image of $\Gamma^{-1}(\theta)$ acting on $H$, denoted by $\Gamma^{-1}(H)$, is a well-defined matrix. If $H+\ell I$ is invertible for all integers $\ell \geq 0$, then the 
reciprocal gamma function [15] is defined by $\Gamma^{-1}(H)=(H)_{\ell} \Gamma^{-1}(H+\ell I)$, where $(H)_{\ell}$ is the shifted factorial matrix function for $H \in \mathbb{C}^{r \times r}$ given as [16]

$$
(H)_{\ell}= \begin{cases}I, & \ell=0 \\ H(H+I), \ldots,(H+(\ell-1) I), & \ell \geq 1 .\end{cases}
$$

$I$ being the $r$-square identity matrix. If $H \in \mathbb{C}^{r \times r}$ is a positive stable matrix and $\ell \geq 1$, then by [15], we have $\Gamma(H)=\lim _{\ell \longrightarrow \infty}(\ell-1) !(H)_{\ell}^{-1} \ell^{H}$.
The Gauss hypergeometric matrix function [16] is defined by

$$
{ }_{2} F_{1}(A, B ; C ; x)=\sum_{n=0}^{\infty} \frac{(A)_{n}(B)_{n}(C)_{n}^{-1}}{n !} x^{n},
$$

for matrices $A, B$, and $C$ in $\mathbb{C}^{r \times r}$, such that $C+k I$ is invertible for all $k \geq 0$ and $|x| \leq 1$.

The Appell matrix functions are defined by

$$
\begin{gathered}
F_{1}\left(A, B, B^{\prime} ; C ; x, y\right)=\sum_{m, n=0}^{\infty} \frac{(A)_{m+n}(B)_{m}\left(B^{\prime}\right)_{n}(C)_{m+n}^{-1}}{m ! n !} x^{m} y^{n}, \\
F_{2}\left(A, B, B^{\prime} ; C, C^{\prime} ; x, y\right)=\sum_{m, n=0}^{\infty} \frac{(A)_{m+n}(B)_{m}\left(B^{\prime}\right)_{n}(C)_{m}^{-1}\left(C^{\prime}\right)_{n}^{-1}}{m ! n !} x^{m} y^{n}, \\
F_{3}\left(A, A^{\prime}, B, B^{\prime} ; C ; x, y\right)=\sum_{m, n=0}^{\infty} \frac{(A)_{m}\left(A^{\prime}\right)_{n}(B)_{m}\left(B^{\prime}\right)_{n}(C)_{m+n}^{-1}}{m ! n !} x^{m} y^{n}, \\
F_{4}\left(A, B ; C, C^{\prime} ; x, y\right)=\sum_{m, n=0}^{\infty} \frac{(A)_{m+n}(B)_{m+n}(C)_{m}^{-1}\left(C^{\prime}\right)_{n}^{-1}}{m ! n !} x^{m} y^{n},
\end{gathered}
$$

where $A, A^{\prime}, B, B^{\prime}, C$, and $C^{\prime}$ are the positive stable matrices in $\mathbb{C}^{r \times r}$, so that $C+k I$ and $C^{\prime}+k I$ are invertible for each integer $k \geq 0$. For regions of convergence of equations (3)-(6), see [17-19].
The Kampé de Fériet hypergeometric matrix function is given as $[18,19]$

$$
F_{m_{2} ; n_{2}, n_{2}^{\prime}}^{m_{1} ; n_{1}, n_{1}^{\prime}}\left(\begin{array}{l}
A: B, C \\
D: E, F
\end{array} ; x, y\right)=\sum_{m, n \geq 0} \prod_{i=1}^{m_{1}}\left(A_{i}\right)_{m+n} \prod_{i=1}^{n_{1}}\left(B_{i}\right)_{m} \prod_{i=1}^{n_{1}^{\prime}}\left(C_{i}\right)_{n} \prod_{i=1}^{m_{2}}\left(D_{i}\right)_{m+n}^{-1} \prod_{i=1}^{n_{2}}\left(E_{i}\right)_{m}^{-1} \prod_{i=1}^{n_{2}^{\prime}}\left(F_{i}\right)_{n}^{-1} \frac{x^{m} y^{n}}{m ! n !},
$$

where $A$ abbreviates the sequence of matrices $A_{1}, \ldots, A_{m_{1}}$, and $A_{i}, B_{i}, C_{i}, D_{i}, E_{i}$, and $F_{i}$ are the positive stable matrices in $\mathbb{C}^{r \times r}$, such that $D_{i}+k I, E_{i}+k I$ and $F_{i}+k I$ are invertible for all integers $k \geq 0$.

Next, we recall the definition of the derivative operator

$$
D_{y} f(y)=\lim _{h \longrightarrow 0} \frac{f(y+h)-f(y)}{h},
$$

provided $f$ is differentiable at $y$. Also, $D_{y}^{k} f(y)=D_{y}\left(D_{y}^{k-1} f(y)\right)$ for $k=0,1,2, \ldots$

In the whole study, $I$ is the identity matrix and $s$ is a nonnegative integer. In the sequel, consider

$$
\begin{aligned}
A+s I & =A_{1}+s I, A_{2}+s I, \ldots, A_{m_{1}}+s I, \\
A^{i} & =A_{1}, \ldots, A_{i-1}, A_{i+1}, \ldots, A_{m_{1}}, \\
A^{i}+s I & =A_{1}+s I, \ldots, A_{i-1}+s I, A_{i+1}+s I, \ldots, A_{m_{1}}+s I .
\end{aligned}
$$

Also, we denote

$$
\begin{gathered}
{[A+k I]_{s}=\prod_{i=1}^{m_{1}}\left(A_{i}+k I\right)_{s},} \\
{[A+k I]_{s}^{-1}=\prod_{i=1}^{m_{1}}\left(A_{i}+k I\right)_{s}^{-1},} \\
{\left[A^{j}+k I\right]_{s}=\prod_{i=1, i \neq j}^{m_{1}}\left(A_{i}+k I\right)_{s},} \\
{\left[A^{j}+k I\right]_{s}^{-1}=\prod_{i=1, i \neq j}^{m_{1}}\left(A_{i}+k I\right)_{s}^{-1} .}
\end{gathered}
$$

\section{Recursion Formulas for the Kampé de Fériet Hypergeometric Matrix Function (K de FHMF)}

In this section, we obtain the recursion formulas for the ( $\mathrm{K}$ de FHMF). 
Theorem 1. Let $A_{i}+s I, i=1, \ldots, m_{1}$ be invertible for each integer $s \geq 0$. Then, the following recursion formula holds true for the ( $K$ de FHMF):

$$
\begin{aligned}
F_{m_{2} ; n_{2}, n_{2}^{\prime}}^{m_{1} ; n_{1}, n_{1}^{\prime}} & \left(\begin{array}{l}
A^{i}, A_{i}+s I: B, C \\
D: E, F
\end{array} ; x, y\right) \\
= & F_{m_{2} ; n_{2}, n_{2}^{\prime}}^{m_{1} ; n_{1}, n_{1}^{\prime}}\left(\begin{array}{l}
A: B, C \\
D: E, F
\end{array} ; x, y\right)+x\left[A^{i}\right][B]\left[\sum_{k=1}^{s} F_{m_{2} ; n_{2}, n_{2}^{\prime}}^{m_{1} ; n_{1}, n_{1}^{\prime}}\left(\begin{array}{l}
A^{i}+I, A_{i}+k I: B+I, C \\
D+I: E+I, F
\end{array} x, y\right)\right][D]^{-1}[E]^{-1} \\
& +y\left[A^{i}\right][C]\left[\sum_{k=1}^{s} F_{m_{2} ; n_{2}, n_{2}^{\prime}}^{m_{1} ; n_{1}, n_{1}^{\prime}}\left(\begin{array}{l}
A^{i}+I, A_{i}+k I: B, C+I \\
D+I: E, F+I
\end{array} x, y\right)\right][D]^{-1}[F]^{-1} .
\end{aligned}
$$

Also, if $A_{i}-k I$ is invertible for integers $k \leq s$, then

$$
\begin{aligned}
& F_{m_{2} ; n_{2}, n_{2}^{\prime}}^{m_{1} ; n_{1}, n_{1}^{\prime}}\left(\begin{array}{l}
A^{i}, A_{i}-s I: B, C \\
D: E, F
\end{array} ; x, y\right) \\
& =F_{m_{2} ; n_{2}, n_{2}^{\prime}}^{m_{1} ; n_{1}, n_{1}^{\prime}}\left(\begin{array}{l}
A: B, C \\
D: E, F
\end{array} ; x, y\right)-x\left[A^{i}\right][B] \sum_{k=0}^{s-1} F_{m_{2} ; n_{2}, n_{2}^{\prime}}^{m_{1} ; n_{1}, n_{1}^{\prime}}\left(\begin{array}{l}
A^{i}+I, A_{i}-k I: B+I, C \\
D+I: E+I, F
\end{array} x, y\right)[D]^{-1}[E]^{-1} \\
& -y\left[A^{i}\right][C] \sum_{k=0}^{s-1} F_{m_{2} ; n_{2}, n_{2}^{\prime}}^{m_{1} ; n_{1}, n_{1}^{\prime}}\left(\begin{array}{l}
A^{i}+I, A_{i}-k I: B, C+I \\
D+I: E, F+I
\end{array} x, y\right)[D]^{-1}[F]^{-1}
\end{aligned}
$$

where $A_{i}, B_{i}, C_{i}, D_{i}, E_{i}$, and $F_{i}$ are the positive stable matrices in $\mathbb{C}^{r \times r}$, such that $A_{i} A_{j}=A_{j} A_{i} ; A_{i} B_{j}=B_{j} A_{i} ; A_{i} C_{j}=C_{j} A_{i}$; $B_{i} C_{j}=C_{j} B_{i} ; F_{i} E_{j}=E_{j} F_{i} ; F_{j} D_{i}=D_{i} F_{j}$; and $D_{i} E_{j}=E_{j} D_{i}$, and $D_{i}+k I, E_{i}+k I$, and $F_{i}+k I$ are invertible for each integer $k \geq 0$.
Proof. In view of equation (7) and the fact that

$$
\left(A_{i}+I\right)_{m+n}=A_{i}^{-1}\left(A_{i}\right)_{m+n}\left(A_{i}+m I+n I\right)
$$

we get the following contiguous matrix relation:

$$
\begin{aligned}
& F_{m_{2} ; n_{2}, n_{2}^{\prime}}^{m_{1} ; n_{1}, n_{1}^{\prime}}\left(\begin{array}{l}
A^{i}, A_{i}+I: B, C \\
D: E, F
\end{array} ; x, y\right) \\
& =F_{m_{2} ; n_{2}, n_{2}^{\prime}}^{m_{1} ; n_{1}, n_{1}^{\prime}}\left(\begin{array}{l}
A: B, C \\
D: E, F
\end{array} x, y\right)+x\left[A^{i}\right][B]\left[F_{m_{2} ; n_{2}, n_{2}^{\prime}}^{m_{1} ; n_{1}, n_{1}^{\prime}}\left(\begin{array}{l}
A+I: B+I, C \\
D+I: E+I, F
\end{array} x, y\right)\right][D]^{-1}[E]^{-1} \\
& +y\left[A^{i}\right][C]\left[F_{m_{2} ; n_{2}, n_{2}^{\prime}}^{m_{1} ; n_{1}, n_{1}^{\prime}}\left(\begin{array}{l}
A+I: B, C+I \\
D+I: E, F+I
\end{array} x, y\right)\right][D]^{-1}[F]^{-1} \text {. }
\end{aligned}
$$

Replacing $A_{i}$ with $A_{i}+I$ in equation (14), we have the following contiguous matrix relation:

$$
\begin{aligned}
& F_{m_{2} ; n_{2}, n_{2}^{\prime}}^{m_{1} ; n_{1}, n_{1}^{\prime}}\left(\begin{array}{l}
A^{i}, A_{i}+2 I: B, C \\
D: E, F
\end{array} ; x, y\right) \\
& \left.=F_{m_{2} ; n_{2}, n_{2}^{\prime}}^{m_{1} ; n_{1}, n_{1}^{\prime}}\left(\begin{array}{l}
A: B, C \\
D: E, F
\end{array} ; x, y\right)+x\left[A^{i}\right][B]\left[\sum_{k=1}^{2} F_{\begin{array}{l}
m_{1} ; n_{2}, n_{2}, n_{2}^{\prime} \\
m_{2}
\end{array}}^{A^{i}+I, A_{i}+k I: B+I, C} ; x, y\right)\right][D]^{-1}[E]^{-1} \\
& \quad+y\left[A^{i}\right][C]\left[\sum_{k=1}^{2} F_{m_{2} ; n_{2}, n_{2}^{\prime}}^{m_{1} ; n_{1}, n_{1}^{\prime}}\left(\begin{array}{l}
A^{i}+I, A_{i}+k I: B, C+I \\
D+I: E, F+I
\end{array} ; x, y\right)\right][D]^{-1}[F]^{-1}
\end{aligned}
$$


Iterating this process $s$-times, we get equation (11). For the proof of equation (12), replace the matrix $A_{i}$ with $A_{i}-I$ in equation (14). As $A_{i}-I$ is invertible, we have

$$
\begin{aligned}
& F_{m_{2} ; n_{2}, n_{2}^{\prime}}^{m_{1} ; n_{1}, n_{1}^{\prime}}\left(\begin{array}{l}
A^{i}, A_{i}-I: B, C \\
D: E, F
\end{array} ; x, y\right)
\end{aligned}
$$

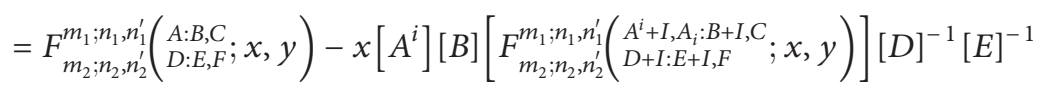

$$
\begin{aligned}
& -y\left[A^{i}\right][C]\left[F_{m_{2} ; n_{2}, n_{2}^{\prime}}^{m_{1} ; n_{1}, n_{1}^{\prime}}\left(\begin{array}{l}
A^{i}+I, A_{i}: B, C+I \\
D+I: E, F+I
\end{array} x, y\right)\right][D]^{-1}[F]^{-1} \text {. }
\end{aligned}
$$

Iteratively, we get equation (12).

Using contiguous matrix relations equations (14) and (16), we get the following forms of the recursion formulas for the (K de FHMF).

Theorem 2. Let $A_{i}+s I, i=1, \ldots, m_{1}$ be invertible for each integer $s \geq 0$. Then, the following recursion formula holds true for the ( $K$ de FHMF):

$$
\begin{aligned}
F_{m_{2} ; n_{2}, n_{2}^{\prime}}^{m_{1} ; n_{1}, n_{1}^{\prime}}\left(\begin{array}{l}
A^{i}, A_{i}+s I: B, C \\
D: E, F
\end{array}\right. & =\sum_{k_{1}+k_{2} \leq s}\left(\begin{array}{c}
s \\
k_{1}, k_{2}
\end{array}\right)\left[A^{i}\right]_{k_{1}+k_{2}}[B]_{k_{1}}[C]_{k_{2}} \\
\quad & \times x^{k_{1}} y^{k_{2}} F_{m_{2} ; n_{2}, n_{2}^{\prime}}^{m_{1} ; n_{1}, n_{1}^{\prime}}\left(\begin{array}{l}
A+\left(k_{1}+k_{2}\right) I: B+k_{1} I, C+k_{2} I \\
D+\left(k_{1}+k_{2}\right) I: E+k_{1} I, F+k_{2} I
\end{array} x, y\right)[D]_{k_{1}+k_{2}}^{-1}[E]_{k_{1}}^{-1}[F]_{k_{2}}^{-1}
\end{aligned}
$$

Also, if $A_{i}-k I$ is invertible for integers $k \leq s$, one writes

$$
\begin{aligned}
& F_{m_{2} ; n_{2}, n_{2}^{\prime}}^{m_{1} ; n_{1}, n_{1}^{\prime}}\left(\begin{array}{l}
A^{i}, A_{i}-s I: B, C \\
D: E, F
\end{array}\right. \\
& =\sum_{k_{1}+k_{2} \leq s}\left(\begin{array}{c}
s \\
k_{1}, k_{2}
\end{array}\right)\left[A^{i}\right]_{k_{1}+k_{2}}[B]_{k_{1}}[C]_{k_{2}} \\
& \quad \times(-x)^{k_{1}}(-y)^{k_{2}} F_{m_{2} ; n_{2}, n_{2}^{\prime}}^{m_{1} ; n_{1}, n_{1}^{\prime}}\left(\begin{array}{l}
A^{i}+\left(k_{1}+k_{2}\right) I, A_{i}: B+k_{1} I, C+k_{2} I \\
D+\left(k_{1}+k_{2}\right) I: E+k_{1} I, F+k_{2} I
\end{array} ; x, y\right)[D]_{k_{1}+k_{2}}^{-1}[E]_{k_{1}}^{-1}[F]_{k_{2}}^{-1},
\end{aligned}
$$

where $A_{i}, B_{i}, C_{i}, D_{i}, E_{i}$, and $F_{i}$ are the positive stable matrices in $\mathbb{C}^{r \times r}$, such that $A_{i} A_{j}=A_{j} A_{i} ; A_{i} B_{j}=B_{j} A_{i} ; A_{i} C_{j}=C_{j} A_{i}$; $B_{i} C_{j}=C_{j} B_{i} ; F_{i} E_{j}=E_{j} F_{i} ; F_{j} D_{i}=D_{i} F_{j}$; and $D_{i} E_{j}=E_{j} D_{i}$, and $D_{i}+k I, E_{i}+k I$, and $F_{i}+k I$ are invertible for each integer $k \geq 0$.

Proof. We prove equation (17) by applying a mathematical induction on $s$. For $s=1$, the result equation (17) is true due to equation (14). Assume equation (17) is true for $s=t$, that is,

$$
\begin{aligned}
& F_{m_{2} ; n_{2}, n_{2}^{\prime}}^{m_{1} ; n_{1}, n_{1}^{\prime}}(\begin{array}{l}
A^{i}, A_{i}+t I: B, C \\
D: E, F
\end{array} \underbrace{\prime} ; x, y) \\
& =\sum_{k_{1}+k_{2} \leq t}\left(\begin{array}{c}
t \\
k_{1}, k_{2}
\end{array}\right)\left[A^{i}\right]_{k_{1}+k_{2}}[B]_{k_{1}}[C]_{k_{2}} \\
& \quad \times x^{k_{1}} y^{k_{2}} F_{m_{2} ; n_{2}, n_{2}^{\prime}}^{m_{1} ; n_{1}, n_{1}^{\prime}}\left(\begin{array}{l}
A+\left(k_{1}+k_{2}\right) I: B+k_{1} I, C+k_{2} I \\
D+\left(k_{1}+k_{2}\right) I: E+k_{1} I, F+k_{2} I
\end{array} x, y\right)[D]_{k_{1}+k_{2}}^{-1}[E]_{k_{1}}^{-1}[F]_{k_{2}}^{-1} .
\end{aligned}
$$

Replacing $A_{i}$ with $A_{i}+I$ in equation (19) and using the contiguous matrix relation equation (14), we get

$$
\begin{aligned}
& F_{m_{2} ; n_{2}, n_{2}^{\prime}}^{m_{1}: n_{1}, n_{1}^{\prime}}\left(\begin{array}{l}
A^{i}, A_{i}+t I+I: B, C \\
D: E, F
\end{array} ; x, y\right) \\
& =\sum_{k_{1}+k_{2} \leq t}\left(\begin{array}{c}
t \\
k_{1}, k_{2}
\end{array}\right)\left[A^{i}\right]_{k_{1}+k_{2}}[B]_{k_{1}}[C]_{k_{2}} x^{k_{1}} y^{k_{2}} \\
& F_{m_{2} ; n_{2}, n_{2}^{\prime}}^{m_{1} ; n_{1}, n_{1}^{\prime}}\left(\begin{array}{l}
A+\left(k_{1}+k_{2}\right) I: B+k_{1} I, C+k_{2} I \\
D+\left(k_{1}+k_{2}\right) I: E+k_{1} I, F+k_{2} I
\end{array} ; x, y\right)[D]_{k_{1}+k_{2}}^{-1}[E]_{k_{1}}^{-1}[F]_{k_{2}}^{-1} \\
& +\sum_{k_{1}+k_{2} \leq t+1}\left(\begin{array}{c}
t \\
k_{1}-1, k_{2}
\end{array}\right)\left[A^{i}\right]_{k_{1}+k_{2}}[B]_{k_{1}}[C]_{k_{2}} x^{k_{1}} y^{k_{2}} \\
& \times F_{m_{2} ; n_{2}, n_{2}^{\prime}}^{m_{1} ; n_{1}, n_{1}^{\prime}}\left(\begin{array}{l}
A+\left(k_{1}+k_{2}\right) I: B+k_{1} I, C+k_{2} I \\
D+\left(k_{1}+k_{2}\right) I: E+k_{1} I, F+k_{2} I
\end{array} ; x, y\right)[D]_{k_{1}+k_{2}}^{-1}[E]_{k_{1}}^{-1}[F]_{k_{2}}^{-1} \\
& +\sum_{k_{1}+k_{2} \leq t+1}\left(\begin{array}{c}
t \\
k_{1}, k_{2}-1
\end{array}\right)\left[A^{i}\right]_{k_{1}+k_{2}}[B]_{k_{1}}[C]_{k_{2}} x^{k_{1}} y^{k_{2}} \\
& \times F_{m_{2} ; n_{2}, n_{2}^{\prime}}^{m_{1} ; n_{1}, n_{1}^{\prime}}\left(\begin{array}{l}
A+\left(k_{1}+k_{2}\right) I: B+k_{1} I, C+k_{2} I \\
D+\left(k_{1}+k_{2}\right) I: E+k_{1} I, F+k_{2} I
\end{array} ; x, y\right)[D]_{k_{1}+k_{2}}^{-1}[E]_{k_{1}}^{-1}[F]_{k_{2}}^{-1} \text {. }
\end{aligned}
$$

Applying the known relation $\left(\begin{array}{l}n \\ k\end{array}\right)+\left(\begin{array}{c}n \\ k-1\end{array}\right)=\left(\begin{array}{c}n+1 \\ k\end{array}\right)$ and $\left(\begin{array}{l}n \\ k\end{array}\right)=0$ (for $k>n$ or $k<0$ ), the above identity can be reduced to the following result: 


$$
\begin{aligned}
& F_{m_{2} ; n_{2}, n_{2}^{\prime}}^{m_{1} ; n_{1}, n_{1}^{\prime}}\left(\begin{array}{l}
A^{i}, A_{i}+t I+I: B, C \\
D: E, F
\end{array}\right. \\
& =\sum_{k_{1}+k_{2} \leq t+1}\left(\begin{array}{c}
t+1 \\
k_{1}, k_{2}
\end{array}\right)\left[A^{i}\right]_{k_{1}+k_{2}}[B]_{k_{1}}[C]_{k_{2}} \\
& \quad \times x^{k_{1}} y^{k_{2}} F_{m_{2} ; n_{2}, n_{2}^{\prime}}^{m_{1} ; n_{1}, n_{1}^{\prime}}\left(\begin{array}{l}
A+\left(k_{1}+k_{2}\right) I: B+k_{1} I, C+k_{2} I \\
D+\left(k_{1}+k_{2}\right) I: E+k_{1} I, F+k_{2} I
\end{array} ; x, y\right)[D]_{k_{1}+k_{2}}^{-1}[E]_{k_{1}}^{-1}[F]_{k_{2}}^{-1} .
\end{aligned}
$$

This establishes equation (17) for $s=t+1$. Hence, the result equation (17) is true for all values of $s$. The second recursion formula (18) is proved in a similar manner.

Now, we present the recursion formulas for matrices $B_{i}$ and $C_{i}$ of the ( $\mathrm{K}$ de FHMF). The proofs of the following results are omitted.

Theorem 3. Let $B_{i}+s I, i=1, \ldots, n_{1}$ be invertible for each integer $s \geq 0$. Then, the following recursion formula is satisfied for the ( $K$ de FHMF):

$$
\begin{aligned}
& F_{m_{2} ; n_{2}, n_{2}^{\prime}}^{m_{1} ; n_{1}, n_{1}^{\prime}}\left(\begin{array}{l}
A: B^{i}, B_{i}+s I, C \\
D: E, F
\end{array}{ }^{=} F_{m_{2} ; n_{2}, n_{2}^{\prime}}^{m_{1} ; n_{1}, n_{1}^{\prime}}\left(\begin{array}{l}
A: B, C \\
D: E, F
\end{array} ; x, y\right)\right. \\
& \quad+x[A]\left[B^{i}\right] \sum_{k=1}^{s} F_{m_{2} ; n_{2}, n_{2}^{\prime}}^{m_{1} ; n_{1}, n_{1}^{\prime}}\left(\begin{array}{l}
A+I: B^{i}+I, B_{i}+k I, C \\
D+I: E+I, F
\end{array}, x, y\right)[D]^{-1}[E]^{-1}
\end{aligned}
$$

In addition, if $B_{i}-k I$ is invertible for integers $k \leq s$, one gets

$$
\begin{aligned}
& F_{m_{2} ; n_{2}, n_{2}^{\prime}}^{m_{1} ; n_{1}, n_{1}^{\prime}}\left(\begin{array}{l}
A: B^{i}, B_{i}-s I, C \\
D: E, F
\end{array} ; x, y\right) \\
& =F_{m_{2} ; n_{2}, n_{2}^{\prime}}^{m_{1} ; n_{1}, n_{1}^{\prime}}\left(\begin{array}{l}
A: B, C \\
D: E, F
\end{array} ; x, y\right) \\
& \quad-x[A]\left[B^{i}\right] \sum_{k=0}^{s-1} F_{m_{2} ; n_{2}, n_{2}^{\prime}}^{m_{1} ; n_{1}, n_{1}^{\prime}}\left(\begin{array}{l}
A+I: B^{i}+I, B_{i}-k I, C \\
D+I: E+I, F
\end{array}, y, y\right)[D]^{-1}[E]^{-1}
\end{aligned}
$$

where $A_{i}, B_{i}, C_{i}, D_{i}, E_{i}$, and $F_{i}$ are the positive stable matrices in $\mathbb{C}^{r \times r}$, such that $A_{i} B_{j}=B_{j} A_{i} ; B_{i} B_{j}=B_{j} B_{i} ; F_{j} D_{i}=D_{i} F_{j}$; $D_{i} E_{j}=E_{j} D_{i}$; and $F_{i} E_{j}=E_{j} F_{i}$, and $D_{i}+k I, E_{i}+k I$, and $F_{i}+$ $k I$ are invertible for each integer $k \geq 0$.

Theorem 4. Let $B_{i}+s I, i=1, \ldots, n_{1}$ be invertible for each integer $s \geq 0$. Then, the following recursion formula is verified for the ( $K$ de FHMF):

$$
\begin{aligned}
F_{m_{2} ; n_{2}, n_{2}^{\prime}}^{m_{1} ; n_{1}, n_{1}^{\prime}}\left(\begin{array}{l}
A: B^{i}, B_{i}+s I, C \\
D: E, F
\end{array} ; x\right)= & \sum_{k=0}^{s}\left(\begin{array}{c}
s \\
k
\end{array}\right)[A]_{k}\left[B^{i}\right]_{k} x^{k} \\
& \times F_{m_{2} ; n_{2}, n_{2}^{\prime}}^{m_{1} ; n_{1}, n_{1}^{\prime}}\left(\begin{array}{l}
A+k I: B+k I: E+k I, F \\
D+k I: E+k, y)[D]_{k}^{-1}[E]_{k}^{-1}
\end{array}\right.
\end{aligned}
$$

Also, if $B_{i}-k I$ is invertible for integers $k \leq s$, we get

$$
\begin{aligned}
F_{m_{2} ; n_{2}, n_{2}^{\prime}}^{m_{1} ; n_{1}, n_{1}^{\prime}}\left(\begin{array}{l}
A: B^{i}, B_{i}-s I, C \\
D: E, F
\end{array} ; x, y\right)= & \sum_{k=0}^{s}\left(\begin{array}{c}
s \\
k
\end{array}\right)[A]_{k}\left[B^{i}\right]_{k}(-x)^{k} \\
& \times F_{m_{2} ; n_{2}, n_{2}^{\prime}}^{m_{1} ; n_{1}, n_{1}^{\prime}}\left(\begin{array}{l}
A+k I: B^{i}+k I, B_{i}, C \\
D+k I: E+k I, F
\end{array} ; x, y\right)[D]_{k}^{-1}[E]_{k}^{-1},
\end{aligned}
$$

where $A_{i}, B_{i}, C_{i}, D_{i}, E_{i}$, and $F_{i}$ are the positive stable matrices in $\mathbb{C}^{r \times r}$, such that $A_{i} B_{j}=B_{j} A_{i} ; B_{i} B_{j}=B_{j} B_{i} ; F_{j} D_{i}=D_{i} F_{j}$; $D_{i} E_{j}=E_{j} D_{i}$; and $F_{i} E_{j}=E_{j} F_{i}$, and $D_{i}+k I, E_{i}+k I$, and $F_{i}+$ $k I$ are invertible for each integer $k \geq 0$.

The recursion formulas for $F_{m_{2} ; n_{2}, n_{2}^{\prime}}^{m_{1} ; n_{1}, n_{1}^{\prime}}\left(\begin{array}{l}A: B, C^{i}, C_{i} \pm s I \\ D: E, F\end{array} ; x, y\right)$ are obtained by replacing $B \leftrightarrow C, E \leftrightarrow F$, and $x \leftrightarrow y$ in Theorems 3 and 4 .
Next, we state the recursion formulas for the matrix $D_{i}$ of the ( $\mathrm{K}$ de FHMF).

Theorem 5. Let $D_{i}-s I, i=1, \ldots, m_{2}$ be invertible for each integer $s \geq 0$. Then, the following recursion formula holds true for the ( $K$ de FHMF):

$$
\begin{aligned}
& F_{m_{2} ; n_{2}, n_{2}^{\prime}}^{m_{1} ; n_{1}, n_{1}^{\prime}}\left(\begin{array}{l}
A: B, C \\
D^{i}, D_{i}-s I: E, F
\end{array} ; x, y\right) \\
& =F_{m_{2} ; n_{2}, n_{2}^{\prime}}^{m_{1} ; n_{1}, n_{1}^{\prime}}\left(\begin{array}{l}
A: B, C \\
D: E, F
\end{array} ; x, y\right) \\
& \quad+x[A][B]\left[\sum_{k=1}^{s} F_{m_{2} ; n_{2}, n_{2}^{\prime}}^{m_{1} ; n_{1}, n_{1}^{\prime}}\left(\begin{array}{l}
A+I: B+I, C \\
D^{i}+I, D_{i}+(2-k) I: E+I, F
\end{array} ;, y\right)\left(D_{i}-k I\right)^{-1}\left(D_{i}-(k-1) I\right)^{-1}\right]\left[D^{i}\right]^{-1}[E]^{-1} \\
& \quad+y[A][C]\left[\sum_{k=1}^{s} F_{m_{2} ; n_{2}, n_{2}^{\prime}}^{m_{1} ; n_{1}, n_{1}^{\prime}}\left(\begin{array}{l}
A+I: B, C+I \\
D^{i}+I, D_{i}+(2-k) I: E, F+I
\end{array} ; x, y\right)\left(D_{i}-k I\right)^{-1}\left(D_{i}-(k-1) I\right)^{-1}\right]\left[D^{i}\right]^{-1}[F]^{-1},
\end{aligned}
$$


where $A_{i}, B_{i}, C_{i}, D_{i}, E_{i}$, and $F_{i}$ are the positive stable matrices in $\mathbb{C}^{r \times r}$, such that $A_{i} B_{j}=B_{j} A_{i} ; A_{i} C_{j}=C_{j} A_{i} ; B_{i} C_{j}=C_{j} B_{i}$; $F_{i} E_{j}=E_{j} F_{i} ; D_{i} D_{j}=D_{j} D_{i} ; F_{j} D_{i}=D_{i} F_{j}$; and $D_{i} E_{j}=E_{j} D_{i}$, and $D_{i}+k I, E_{i}+k I$, and $F_{i}+k I$ are invertible for each integer $k \geq 0$.

Proof. Applying the definition of the (K de FHMF) and the fact that

$$
\left(D_{i}-I\right)_{m+n}^{-1}=\left(D_{i}\right)_{m+n}^{-1}\left[1+m\left(D_{i}-I\right)^{-1}+n\left(D_{i}-I\right)^{-1}\right],
$$

the following contiguous matrix relation is obtained:

$$
\begin{aligned}
F_{m_{2} ; n_{2}, n_{2}^{\prime}}^{m_{1} ; n_{1}, n_{1}^{\prime}}\left(\begin{array}{l}
A: B, C \\
D^{i}, D_{i}-I: E, F
\end{array} ; x, y\right) \\
=F_{m_{2} ; n_{2}, n_{2}^{\prime}}^{m_{1} ; n_{1}, n_{1}^{\prime}}\left(\begin{array}{c}
A: B, C, C \\
D: E, F
\end{array} x, y\right) \\
\quad+x[A][B]\left[F_{m_{2} ; n_{2}, n_{2}^{\prime}}^{m_{1} ; n_{1}, n_{1}^{\prime}}\left(\begin{array}{l}
A+I: B+I, C \\
D+I: E+I, F
\end{array} x, y\right)\left(D_{i}-I\right)^{-1}\right][D]^{-1}[E]^{-1} \\
\quad+y[A][C]\left[F_{m_{2} ; n_{2}, n_{2}^{\prime}}^{m_{1} ; n_{1}, n_{1}^{\prime}}\left(\begin{array}{l}
A+I: B, C+I \\
D+I: E, F+I
\end{array} ; x, y\right)\left(D_{i}-I\right)^{-1}\right][D]^{-1}[F]^{-1}
\end{aligned}
$$

With the help of this contiguous matrix relation to the ( $\mathrm{K}$ de FHMF) with the matrix $D_{i}-s I$ for $s$-times, we get equation (26).

Next, we give recursion formulas for the (K de FHMF) $E_{i}, F_{i}$. The proof is omitted for the following result.

Theorem 6. Let $E_{i}-s I, i=1, \ldots, n_{2}$ be invertible for each integer $s \geq 0$. Then, the following recursion formula holds true for the (K de FHMF):

$$
\begin{aligned}
& F_{m_{2} ; n_{2}, n_{2}^{\prime}}^{m_{1} ; n_{1}, n_{1}^{\prime}}\left(\begin{array}{l}
A: B, C \\
D: E^{i}, E_{i}-s I, F
\end{array} ; x, y\right) \\
& =F_{m_{2} ; n_{2}, n_{2}^{\prime}}^{m_{1} ; n_{1}, n_{1}^{\prime}}\left(\begin{array}{l}
A: B, C, F \\
D: E, y
\end{array}\right) \\
& +x[A][B]\left[\sum_{k=1}^{s} F_{m_{2} ; n_{2}, n_{2}^{\prime}}^{m_{1} ; n_{1}, n_{1}^{\prime}}\left(\begin{array}{l}
A+I: B+I, C \\
D+I: E^{i}+I, E_{i}+(2-k) I, F
\end{array} ; x, y\right)\left(E_{i}-k I\right)^{-1}\left(E_{i}-(k-1) I\right)^{-1}\right]\left[E^{i}\right]^{-1}[D]^{-1},
\end{aligned}
$$

where $A_{i}, B_{i}, C_{i}, D_{i}, E_{i}$, and $F_{i}$ are the positive stable matrices in $\mathbb{C}^{r \times r}$, such that $A_{i} B_{j}=B_{j} A_{i} ; E_{i} E_{j}=E_{j} E_{i} ; F_{i} E_{j}=E_{j} F_{i}$; $F_{j} D_{j}=D_{j} F_{i}$; and $D_{j} E_{i}=E_{i} D_{j}$, and $D_{i}+k I, E_{i}+k I$, and $F_{i}+k I$ are invertible for each integer $k \geq 0$.

The recursion formulas for $F_{m_{2} ; n_{2}, n_{2}^{\prime}}^{m_{1} ; n_{1}, n_{1}^{\prime}}\left(\begin{array}{l}A: B, C, F^{i}, F_{i}-s I \\ D\end{array} ; x, y\right)$ are obtained by replacing $B \leftrightarrow C, E \leftrightarrow F$, and $x \leftrightarrow y$ in Theorem 6 .

\section{Finite Matrix Summation Formulas for the Kampé de Fériet Hypergeometric Matrix Function by a Derivative Operator}

In this section, we obtain the finite matrix summation formulas for the ( $\mathrm{K}$ de $\mathrm{FHMF}$ ) by a derivative operator. These formulas are matrix analogues for some summation formulas of double hypergeometric functions [8]. The $p^{\text {th }}$ derivative on $y$ of the (K de FHMF) is obtained as follows:

$$
\begin{aligned}
D_{y}^{p} & \left\{F_{m_{2} ; n_{2}, n_{2}^{\prime}}^{m_{1} ; n_{1}, n_{1}^{\prime}}\left(\begin{array}{l}
A: B, C \\
D: E, F
\end{array} ; x, y\right)\right\} \\
& =[A]_{p}[C]_{p} F_{m_{2} ; n_{2}, n_{2}^{\prime}}^{m_{1} ; n_{1}, n_{1}^{\prime}}\left(\begin{array}{l}
A+p I: B, C+p I \\
D+p I: E, F+p I
\end{array} x, y\right)[D]_{p}^{-1}[F]_{p}^{-1},
\end{aligned}
$$

where $A_{i}, B_{i}, C_{i}, D_{i}, E_{i}$, and $F_{i}$ are the positive stable matrices in $\mathbb{C}^{r \times r}$, such that $A_{i} C_{j}=C_{j} A_{i} ; B_{i} C_{j}=C_{j} B_{i} ; D_{i} E_{j}=E_{j} D_{i}$; and $D_{i} F_{j}=F_{j} D_{i}$, and $D_{i}+k I, E_{i}+k I$, and $F_{i}+k I$ are invertible for each integer $k \geq 0$.

By using the generalized Leibnitz formula,

$$
D_{y}^{p}(f(y) g(y))=\sum_{k=0}^{p}\left(\begin{array}{l}
p \\
k
\end{array}\right) D_{y}^{p-k} f(y) D_{y}^{k} g(y),
$$

and equation (30), we derive the following finite matrix summation formulas of the (K de FHMF).

Theorem 7. Let $A_{i}, B_{i}, C_{i}, D_{i}, E_{i}$, and $F_{i}$ be the positive stable matrices in $\mathbb{C}^{r \times r}$, such that $A_{i} C_{j}=C_{j} A_{i} ; B_{i} C_{j}=C_{j} B_{i}$; $C_{i} C_{j}=C_{j} C_{i} ; D_{i} E_{j}=E_{j} D_{i} ;$ and $D_{i} F_{j}=F_{j} D_{i}$, and $D_{i}+k I$, $E_{i}+k I$, and $F_{i}+k I$ are invertible for all integers $k \geq 0$. Then, the following finite matrix summation formulas hold for the ( $K$ de FHMF):

$$
\begin{aligned}
& \sum_{k=0}^{p}\left(\begin{array}{c}
p \\
k
\end{array}\right)[A]_{k}\left[C^{i}\right]_{k} y^{k} F_{m_{2} ; n_{2}, n_{2}^{\prime}}^{m_{1} ; n_{1}, n_{1}^{\prime}}\left(\begin{array}{l}
A+k I: B, C+k I \\
D+k I: E, F+k I
\end{array} ; x, y\right)[D]_{k}^{-1}[F]_{k}^{-1} \\
& \quad=F_{m_{2} ; n_{2}, n_{2}^{\prime}}^{m_{1} ; n_{1}, n_{1}^{\prime}}(\begin{array}{l}
A: B, C^{i}, C_{i}+p I \\
D: E, F
\end{array} \underbrace{-1} y) .
\end{aligned}
$$

Proof. From definition of the (K de FHMF) and the generalized Leibnitz formula for differentiation of a product of two functions, we have 


$$
\begin{aligned}
D_{y}^{p}\left\{y^{C_{i}+p I-I} F_{m_{2} ; n_{2}, n_{2}^{\prime}}^{m_{1} ; n_{1}, n_{1}^{\prime}}\left(\begin{array}{l}
A: B, C, F \\
D: E, F
\end{array} x, y\right)\right\} \\
=\sum_{k=0}^{p}\left(\begin{array}{c}
p \\
k
\end{array}\right) D_{y}^{p-k}\left\{y^{C_{i}+p I-I}\right\} D_{y}^{k}\left\{F_{m_{2} ; n_{2}, n_{2}^{\prime}}^{m_{1} ; n_{1}, n_{1}^{\prime}}\left(\begin{array}{l}
A: B, C \\
D: E, F
\end{array} ; x, y\right)\right\} \\
=\left(C_{i}\right)_{p} y^{C_{i}-I} \sum_{k=0}^{p}\left(\begin{array}{c}
p \\
k
\end{array}\right)[A]_{k}\left[C^{i}\right]_{k} \\
\quad \times y^{k} F_{m_{2} ; n_{2}, n_{2}^{\prime}}^{m_{1} ; n_{1}, n_{1}^{\prime}}\left(\begin{array}{l}
A+k I: B, C+k I \\
D+k I: E, F+k I
\end{array} x, y\right)[D]_{k}^{-1}[F]_{k}^{-1} .
\end{aligned}
$$

We used equation (30) and some simplification in the second equality. Next, we combine $y^{C_{i}+(p-1) I}$ with the variable $y$ in the (K de FHMF) and apply the derivative operator $p$-times on $y$ to get the following result:

$$
\begin{aligned}
& D_{y}^{p}\left\{y^{C_{i}+(p-1) I} F_{m_{2} ; n_{2}, n_{2}^{\prime}}^{m_{1} ; n_{1}, n_{1}^{\prime}}\left(\begin{array}{l}
A: B, C, C \\
D: E, x
\end{array} ;\right)\right\} \\
& =\sum_{m, n=0}^{\infty}\left(C_{i}+n I\right)_{p} \prod_{i=1}^{m_{1}}\left(A_{i}\right)_{m+n} \prod_{i=1}^{n_{1}}\left(B_{i}\right)_{m} \prod_{i=1}^{n_{1}^{\prime}}\left(C_{i}\right)_{n} \prod_{i=1}^{m_{2}}\left(D_{i}\right)_{m+n}^{-1} \prod_{i=1}^{n_{2}}\left(E_{i}\right)_{m}^{-1} \prod_{i=1}^{n_{2}^{\prime}}\left(F_{i}\right)_{n}^{-1} \\
& \times \frac{x^{m} y^{C_{i}+(n-1) I}}{m ! n !}=\left(C_{i}\right)_{p} y^{C_{i}-I} F_{m_{2} ; n_{2}, n_{2}^{\prime}}^{m_{1} ; n_{1}, n_{1}^{\prime}}\left(\begin{array}{l}
A: B, C_{i}+p I, C^{i} \\
D: E, F
\end{array} ; x, y\right) .
\end{aligned}
$$

Equating the above two relations leads to $D_{i} E_{j}=E_{j} D_{i} ; D_{i} F_{j}=F_{j} D_{i}$; and $F_{i} F_{j}=F_{j} F_{i}$, and $D_{i}+k I$, equation (32).

Theorem 8. Let $A_{i}, B_{i}, C_{i}, D_{i}, E_{i}$, and $F_{i}$ be the positive stable $E_{i}+k I$, and $F_{i}+k I$ are invertible for all integers $k \geq 0$. Then, the following finite matrix summation formulas of the ( $K$ de matrices in $\mathbb{C}^{r \times r}$, such that $A_{i} C_{j}=C_{j} A_{i} ; B_{i} C_{j}=C_{j} B_{i}$; FHMF) hold true:

$$
\begin{aligned}
& \sum_{k=0}^{p}\left(\begin{array}{c}
p \\
k
\end{array}\right)[A]_{k}[C]_{k} y^{k} F_{m_{2} ; n_{2}, n_{2}^{\prime}}^{m_{1} ; n_{1}, n_{1}^{\prime}}\left(\begin{array}{l}
A+k I: B, C+k I \\
D+k I: E, F+k I
\end{array} ; x, y\right)\left(F_{i}-p I\right)_{k}^{-1}[D]_{k}^{-1}[F]_{k}^{-1} \\
& \quad=F_{m_{2} ; n_{2}, n_{2}^{\prime}}^{m_{1} ; n_{1}, n_{1}^{\prime}}\left(\begin{array}{l}
A: B, C \\
D: E, F_{i}-p I, F^{i}
\end{array} ; x, y\right),
\end{aligned}
$$

where $F_{i}+(k-p) I$ is an invertible matrix for $0 \leq k \leq p$ and $i=1, \ldots, n_{2}^{\prime}$.

Proof. Applying the derivative operator on $F_{m_{2} ; n_{2}, n_{2}^{\prime}}^{m_{1} ; n_{1}, n_{1}^{\prime}}\left(\begin{array}{l}A: B, C \\ D: E, F\end{array} x, y\right) y^{F_{i}-I}, p$-times, gives the formula as explained in the proof of Theorem 7 . We omit the details.
Applying a derivative operator and some transformations, we can get the finite matrix summation formulas of the ( $\mathrm{K}$ de FHMF) as follows.

Theorem 9. Let $A_{i}, B_{i}, C_{i}, D_{i}, E_{i}$, and $F_{i}$ be the positive stable matrices in $\mathbb{C}^{r \times r}$, such that $A_{i} C_{j}=C_{j} A_{i} ; B_{i} C_{j}=C_{j} B_{i}$; 
$D_{i} E_{j}=E_{j} D_{i} ;$ and $D_{i} F_{j}=F_{j} D_{i}$, and $D_{i}+k I, E_{i}+k I$, and $F_{i}+k I$ are invertible for each integer $k \geq 0$. Then, the following finite matrix summation formulas of the ( $K$ de FHMF) hold true:

$$
\begin{aligned}
& \sum_{k=0}^{r}\left(\begin{array}{c}
r \\
k
\end{array}\right)(-1)^{k} F_{m_{2} ; n_{2}, n_{2}^{\prime}}^{m_{1} ; n_{1}, n_{1}^{\prime}}\left(\begin{array}{l}
A: B, C, C, F_{i}-k I, F^{i} \\
D: x, y)\left(I-F_{i}\right)_{k}\left((2-r) I-F_{i}\right)_{k}^{-1}
\end{array}\right. \\
& \quad=(-1)^{r}[A]_{r}[C]_{r} y^{r} F_{m_{2} ; n_{2}, n_{2}^{\prime}}^{m_{1} ; n_{1}, n_{1}^{\prime}}\left(\begin{array}{l}
A+r I: B, C+r I \\
D+r I: E, F+r I
\end{array} ; x, y\right)\left(F_{i}-I\right)_{r}^{-1}[D]_{r}^{-1}[F]_{r}^{-1}, \\
& \sum_{k=0}^{r}\left(\begin{array}{l}
r \\
k
\end{array}\right)(-1)^{k} F_{m_{2} ; n_{2}, n_{2}^{\prime}}^{m_{1} ; n_{1}, n_{1}^{\prime}}\left(\begin{array}{l}
A: B, C \\
D: E, F_{i}+k I, F^{i}
\end{array} ; x, y\right)\left(F_{i}+(r-1) I\right)_{k}\left(F_{i}\right)_{k}^{-1} \\
& \quad=[A]_{r}[C]_{r} y^{r} F_{m_{2} ; n_{2}, n_{2}^{\prime}}^{m_{1} ; n_{1}, n_{1}^{\prime}}\left(\begin{array}{l}
A+r I: B, C+r I \\
D+r I: E, F_{i}+2 r I, F^{i}+r I
\end{array} ; x, y\right)[D]_{r}^{-1}[F]_{r}^{-1}\left(F_{i}+r I\right)_{r}^{-1},
\end{aligned}
$$

where $(2+k-r) I-F_{i}, F_{i}-k I$, and $F_{i}+(k-1) I$ is an invertible matrix for $0 \leq k \leq r$ in (36); $F_{i}+r I$ is an invertible matrix in equation (37), $i=1, \ldots, n_{2}^{\prime}$.
Proof. We first prove identity equation (36). From the definition of the (K de FHMF) and the generalized Leibnitz formula for differentiation of a product of two functions, we obtain the following result:

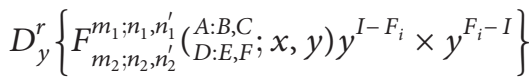

$$
\begin{aligned}
& \left.=\sum_{k=0}^{r}\left(\begin{array}{l}
r \\
k
\end{array}\right) D_{y}^{k}\left\{F_{F_{2} ; n_{2}, n_{2}^{\prime}}^{m_{1} ; n_{1}, n_{1}^{\prime}} \begin{array}{l}
A: B, C \\
D: E, F
\end{array} ; x, y\right) y^{F_{i}-I}\right\} D_{y}^{r-k}\left\{y^{I-F_{i}}\right\} \\
& =\sum_{k=0}^{r}(-1)^{r+k}\left(\begin{array}{l}
r \\
k
\end{array}\right) F_{m_{2} ; n_{2}, n_{2}^{\prime}}^{m_{1} ; n_{1}, n_{1}^{\prime}}\left(\begin{array}{l}
A: B, C \\
D: E, F_{i}-k I, F^{i}
\end{array} ; x, y\right) \\
& \times\left(F_{i}-I\right)_{r}\left(I-F_{i}\right)_{k}\left(2 I-F_{i}-r I\right)_{k}^{-1} y^{-r} \text {. }
\end{aligned}
$$

Now, using the derivative operator on the (K de FHMF) for $r$-times directly and equating with the above equality gives equation (36) after some simplifications. Next, applying the operator $D_{y}^{r}$ on

$$
F_{m_{2} ; n_{2}, n_{2}^{\prime}}^{m_{1} ; n_{1}, n_{1}^{\prime}}\left(\begin{array}{l}
A: B, C \\
D: E, F_{i}+r I, F^{i}
\end{array} ; x, y\right) y^{I-F_{i}-r I} \times y^{F_{i}+I r-I},
$$

and proceeding as in the proof of equation (36) gives the result equation (37).

\section{Infinite Summation Formulas for the Kampé de Fériet Hypergeometric Matrix Function}

In this section, we will establish the infinite summation formulas of the ( $\mathrm{K}$ de FHMF).

Theorem 10. Let $A_{i}, B_{i}, C_{i}, D_{i}, E_{i}$, and $F_{i}$ be the positive stable matrices in $\mathbb{C}^{r \times r}$, such that $D_{i}+k I, E_{i}+k I$, and $F_{i}+k I$ are invertible for each integer $k \geq 0$. Then, the following infinite summation formulas of the ( $K$ de FHMF) hold true:

$$
\begin{aligned}
& \sum_{k=0}^{\infty} \frac{\left(A_{i}\right)_{k}}{k !} t^{k} F^{m_{1} ; n_{1}, n_{1}^{\prime}} \underbrace{}_{m_{2} ; n_{2}, n_{2}^{\prime}}\left(\begin{array}{l}
A_{i}+k I, A^{i}: B, C \\
D: E, F
\end{array} ; x, y\right) \\
& =(1-t)^{-A_{i}} F_{m_{2} ; n_{2}, n_{2}^{\prime}}^{m_{1} ; n_{1}, n_{1}^{\prime}}\left(\begin{array}{l}
A: B, C, C \\
D: E, F
\end{array} \frac{x}{1-t}, \frac{y}{1-t}\right),
\end{aligned}
$$

where $i=1, \ldots, m_{1}$;

$$
\begin{aligned}
& \sum_{k=0}^{\infty} \frac{\left(B_{i}\right)_{k}}{k !} t^{k} F_{m_{2} ; n_{2}, n_{2}^{\prime}}^{m_{1} ; n_{1}, n_{1}^{\prime}}\left(\begin{array}{l}
A: B_{i}+k I, B^{i}, C \\
D: E, F
\end{array} ; x, y\right) \\
& \quad=(1-t)^{-B_{i}} F_{m_{2} ; n_{2}, n_{2}^{\prime}}^{m_{1} ; n_{1}, n_{1}^{\prime}}\left(\begin{array}{l}
A: B, C \\
D: E, F
\end{array} ; \frac{x}{1-t}, y\right), A_{i} B_{j}=B_{j} A_{i},
\end{aligned}
$$

where $i=1, \ldots, n_{1}$.

Proof: We shall prove equation (40). We apply the definition of the (K de FHMF) and transformation,

$$
\left(A_{i}\right)_{k}\left(A_{i}+k I\right)_{m+n}=\left(A_{i}\right)_{m+n}\left(A_{i}+(m+n) I\right)_{k},
$$

to get that the left side of equation (40) is written as 


$$
\sum_{m, n=0}^{\infty}{ }_{1} F_{0}\left[l_{-}^{A_{i}+(m+n) I} ; t\right] \prod_{i=1}^{m_{1}}\left(A_{i}\right)_{m+n} \prod_{i=1}^{n_{1}}\left(B_{i}\right)_{m} \prod_{i=1}^{n_{1}^{\prime}}\left(C_{i}\right)_{n} \prod_{i=1}^{m_{2}}\left(D_{i}\right)_{m+n}^{-1} \prod_{i=1}^{n_{2}}\left(E_{i}\right)_{m}^{-1} \prod_{i=1}^{n_{2}^{\prime}}\left(F_{i}\right)_{n}^{-1} \frac{x^{m} y^{n}}{m ! n !} .
$$

Using the identity,

$$
{ }_{1} F_{0}[A ;-; t]=(1-t)^{-A},
$$

and after simplifications, the right side of equation (40) is obtained. This ends the proof of equation (40). The identity equation (41) is proved in a similar manner.
Theorem 11. Let $A_{i}, B_{i}, C_{i}, D_{i}, E_{i}$, and $F_{i}$ be the positive stable matrices in $\mathbb{C}^{r \times r}$, such that $A_{i} B_{j}=B_{j} A_{i} ; D_{i} E_{j}=E_{j} D_{i}$; $D_{i} F_{j}=F_{j} D_{i}$; and $E_{i} F_{j}=F_{j} E_{i}$, and $D_{i}+k I, E_{i}+k I$, and $F_{i}+$ $k I$ are invertible for each integer $k \geq 0$. Then, the infinite summation formulas of the ( $K$ de FHMF) hold true:

$$
F_{m_{2} ; n_{2}, n_{2}^{\prime}}^{m_{1} ; n_{1}, n_{1}^{\prime}}\left(\begin{array}{l}
A: B, C, C \\
D: E, F
\end{array} ; x+t, y\right)=\sum_{k=0}^{\infty}[A]_{k}[B]_{k} \frac{t^{k}}{k !} \times F_{m_{2} ; n_{2}, n_{2}^{\prime}}^{m_{1} ; n_{1}, n_{1}^{\prime}}\left(\begin{array}{l}
A+k I: B+k I, C \\
D+k I: E+k I, F
\end{array} x, y\right)[D]_{k}^{-1}[E]_{k}^{-1}
$$

Proof: From the definition of the (K de FHMF) and the transformation $(A)_{k}\left((A+k I)_{m}=(A)_{k+m}\right.$, the right side of equation (45) is expressed as

$$
\sum_{k, m, n=0}^{\infty} \prod_{i=1}^{m_{1}}\left(A_{i}\right)_{m+n+k} \prod_{i=1}^{n_{1}}\left(B_{i}\right)_{m+k} \prod_{i=1}^{n_{1}^{\prime}}\left(C_{i}\right)_{n} \prod_{i=1}^{m_{2}}\left(D_{i}\right)_{m+n+k}^{-1} \prod_{i=1}^{n_{2}}\left(E_{i}\right)_{m+k}^{-1} \prod_{i=1}^{n_{2}^{\prime}}\left(F_{i}\right)_{n}^{-1} \frac{x^{m} y^{n}}{m ! n ! k !} t^{k}
$$

Replace $m+k$ by $l$ in the above result. After some simplifications, we have

$$
\begin{gathered}
\sum_{l, n=0}^{\infty} \prod_{i=1}^{m_{1}}\left(A_{i}\right)_{l+n} \prod_{i=1}^{n_{1}}\left(B_{i}\right)_{l} \prod_{i=1}^{n_{1}^{\prime}}\left(C_{i}\right)_{n} \prod_{i=1}^{m_{2}}\left(D_{i}\right)_{l+n}^{-1} \prod_{i=1}^{n_{2}}\left(E_{i}\right)_{l}^{-1} \prod_{i=1}^{n_{2}^{\prime}}\left(F_{i}\right)_{n}^{-1} \frac{y^{n}}{l ! n !} \sum_{k=0}^{l}\left(\begin{array}{l}
l \\
k
\end{array} x^{l-k} t^{k}\right. \\
=\sum_{l, n=0}^{\infty} \prod_{i=1}^{m_{1}}\left(A_{i}\right)_{l+n} \prod_{i=1}^{n_{1}}\left(B_{i}\right)_{l} \prod_{i=1}^{n_{1}^{\prime}}\left(C_{i}\right)_{n} \prod_{i=1}^{m_{2}}\left(D_{i}\right)_{l+n}^{-1} \prod_{i=1}^{n_{2}}\left(E_{i}\right)_{l}^{-1} \prod_{i=1}^{n_{2}^{\prime}}\left(F_{i}\right)_{n}^{-1} \frac{(x+t)^{l} y^{n}}{l ! n !}
\end{gathered}
$$

Using the relation

$$
\sum_{k=0}^{l}\left(\begin{array}{l}
l \\
k
\end{array}\right) x^{k} y^{l-k}=(x+y)^{l}
$$

in the inner summation, finishes the proof of equation (45).

Theorem 12. Let $A_{i}, B_{i}, C_{i}, D_{i}, E_{i}$, and $F_{i}$ be the positive stable matrices in $\mathbb{C}^{r \times r}$, such that $D_{i}+k I, E_{i}+k I$, and $F_{i}+k I$ are invertible for each integer $k \geq 0$. Then, the infinite summation formulas of the ( $K$ de FHMF) hold true:

$$
\begin{gathered}
\sum_{k=0}^{\infty} \frac{\left(B_{i}\right)_{k}}{k !}(-t)^{k} F_{m_{2} ; n_{2}, n_{2}^{\prime}}^{m_{1} ; n_{1}, n_{1}^{\prime}}\left(\begin{array}{l}
A:-k I, B^{i}, C \\
D: E, F
\end{array} ; \frac{1+t}{t} x, y\right) \\
=(1+t)^{-B_{i}} F_{m_{2} ; n_{2}, n_{2}^{\prime}}^{m_{1} ; n_{1}, n_{1}^{\prime}}\left(\begin{array}{l}
A: B, C \\
D: E, F
\end{array} ; x, y\right),
\end{gathered}
$$

where $A_{j} B_{i}=B_{i} A_{j}, i=1, \ldots, n_{1}$. 
Proof: From the definition of the (K de FHMF), the left side of equation (49) can be expressed as

$$
\sum_{k, n=0}^{\infty} \sum_{m=0}^{k} \frac{\left(B_{i}\right)_{k}(-k I)_{m}}{m ! n ! k !} \prod_{i=1}^{m_{1}}\left(A_{i}\right)_{m+n} \prod_{j=1, \neq i}^{n_{1}}\left(B_{i}\right)_{m} \prod_{i=1}^{n_{1}^{\prime}}\left(C_{i}\right)_{n} \prod_{i=1}^{m_{2}}\left(D_{i}\right)_{m+n}^{-1} \prod_{i=1}^{n_{2}}\left(E_{i}\right)_{m}^{-1} \prod_{i=1}^{n_{2}^{\prime}}\left(F_{i}\right)_{n}^{-1}\left(\frac{1+t}{t} x\right)^{m} y^{n}(-t)^{k}
$$

Taking $k=m+l$, changing the summation order and simplifying, we get

$$
\sum_{m, n=0}^{\infty}{ }_{1} F_{0}\left[\left[_{i}^{B_{i}+m I} ;-t\right] \prod_{i=1}^{m_{1}}\left(A_{i}\right)_{m+n} \prod_{i=1}^{n_{1}}\left(B_{i}\right)_{m} \prod_{i=1}^{n_{1}^{\prime}}\left(C_{i}\right)_{n} \prod_{i=1}^{m_{2}}\left(D_{i}\right)_{m+n}^{-1} \prod_{i=1}^{n_{2}}\left(E_{i}\right)_{m}^{-1} \prod_{i=1}^{n_{2}^{\prime}}\left(F_{i}\right)_{n}^{-1} \frac{x^{m} y^{n}}{m ! n !}(1+t)^{m} .\right.
$$

Evaluating the inner ${ }_{1} F_{0}$-series in the above equation by equation (44),

$$
{ }_{1} F_{0}\left[B_{i}+m I ;-;-t\right]=(1+t)^{-B_{i}-m I},
$$

and simplifying, we get the right side of this theorem. This completes the proof.

Theorem 13. Let $A_{i}, B_{i}, C_{i}, D_{i}, E_{i}$, and $F_{i}$ be the positive stable matrices in $\mathbb{C}^{r \times r}$, such that $D_{i}+k I, E_{i}+k I$, and $F_{i}+k I$ are invertible for all integers $k \geq 0$. Then, the infinite summation formulas of the (K de FHMF) hold true:

$$
\begin{aligned}
\sum_{k=0}^{\infty} & \frac{\left(B_{i}\right)_{k}}{k !}\left(\frac{t+x}{x-1}\right)^{k} F_{m_{2} ; n_{2}, n_{2}^{\prime}}^{m_{1} ; n_{1}, n_{1}^{\prime}}\left(\begin{array}{l}
A:-k I, B^{i}, C \\
D: E, F
\end{array} \frac{1+t}{t+x} x, y\right) \\
= & \left(\frac{1-x}{t+1}\right)^{B_{i}} F_{m_{2} ; n_{2}, n_{2}^{\prime}}^{m_{1} ; n_{1}, n_{1}^{\prime}}\left(\begin{array}{l}
A: B, C, E \\
D: E, F
\end{array} x, y\right),
\end{aligned}
$$

where $A_{j} B_{i}=B_{i} A_{j}, i=1, \ldots, n_{1}$.

Proof: It is similar to Theorem 12. We omit the details.

\section{Conclusion}

We have investigated recursion formulas and some finite matrix and infinite matrix summation formulas involving the ( $\mathrm{K}$ de FHMF). We remark that by specializing the sequence of matrices in the ( $\mathrm{K}$ de FHMF), we can deduce recursion formulas and finite matrix and infinite matrix summation formulas for some Appell matrix functions [17-19].

\section{Data Availability}

The data used to support the findings of this study are available from the corresponding author upon request.

\section{Conflicts of Interest}

The authors declare that there are no conflicts of interest.

\section{Authors' Contributions}

All authors contributed equally and significantly in writing this article. All authors read and approved the final manuscript.

\section{References}

[1] S. B. Opps, N. Saad, and H. M. Srivastava, "Recursion formulas for appell's hypergeometric function with some applications to radiation field problems," Applied Mathematics and Computation, vol. 207, no. 2, pp. 545-558, 2009.

[2] X. Wang, "Recursion formulas for appell functions," Integral Transforms and Special Functions, vol. 23, no. 6, pp. 421-433, 2012.

[3] V. Sahai and A. Verma, "Recursion formulas for multivariable hypergeometric functions," Asian-European Journal of Mathematics, vol. 8, no. 4, Article ID 1550082, 2015.

[4] V. Sahai and A. Verma, "Recursion formulas for exton's triple hypergeometric functions," Kyungpook Mathematical Journal, vol. 56, no. 2, pp. 473-506, 2016.

[5] A. Hasanov, J. Younis, and H. Aydi, "On decomposition formulas related to the gaussian hypergeometric functions in three variables," Journal of Function Spaces, vol. 2021, Article ID 5538583, 6 pages, 2021.

[6] V. Sahai and A. Verma, "Recursion formulas for the srivastava-daoust and related multivariable hypergeometric functions," Asian-European Journal of Mathematics, vol. 9, no. 4, Article ID 1650081, 2016.

[7] D. Baleanu and P. Agarwal, "On generalized fractional integral operators and the generalized gauss hypergeometric functions," Abstract and Applied Analysis, vol. 2014, Article ID 630840, 5 pages, 2014.

[8] X. Wang and Y. Chen, "Finite summation formulas of double hypergeometric functions," Integral Transforms and Special Functions, vol. 28, no. 3, pp. 239-253, 2017.

[9] A. G. Constantine and R. J. Muirhead, "Partial differential equations for hypergeometric functions of two argument matrices," Journal of Multivariate Analysis, vol. 2, no. 3, pp. 332-338, 1972.

[10] A. T. James, "Special functions of matrix and single argument in statistics," in Theory and Applications of Special Functions, 
R. A. Askey, Ed., Academic Press, New York, NY, USA, pp. $497-520,1975$.

[11] L. Jódar, R. Company, and E. Navarro, "Laguerre matrix polynomials and systems of second-order differential equations," Applied Numerical Mathematics, vol. 15, no. 1, pp. 53-63, 1994.

[12] L. Jódar, R. Company, and E. Ponsoda, "Orthogonal matrix polynomials and systems of second order differential equations," Differential Equations and Dynamical Systems, vol. 3, pp. 269-288, 1995.

[13] P. O. Mohammed, H. Aydi, A. Kashuri, Y. S. Hamed, and K. M. Abualnaja, "Midpoint inequalities in fractional calculus defined using positive weighted symmetry function kernels," Symmetry, vol. 13, no. 4, p. 550, 2021.

[14] A. Verma, "Some results on the srivastava's triple hypergeometric matrix functions," Asian-European Journal of Mathematics, vol. 14, no. 4, Article ID 2150056, 2020.

[15] L. Jódar and J. C. Cortés, "Some properties of gamma and beta matrix functions," Applied Mathematics Letters, vol. 11, no. 1, pp. 89-93, 1998.

[16] L. Jódar and J. C. Cortés, "On the hypergeometric matrix function," Journal of Computational and Applied Mathematics, vol. 99, no. 1-2, pp. 205-217, 1998.

[17] A. Altin, B. Çekim, and R. Şahin, "On the matrix versions of appell hypergeometric functions," Quaestiones Mathematicae, vol. 37, no. 1, pp. 31-38, 2014.

[18] R. Dwivedi and V. Sahai, "On the hypergeometric matrix functions of two variables," Linear and Multilinear Algebra, vol. 66, no. 9, pp. 1819-1837, 2017.

[19] R. Dwivedi and V. Sahai, "A note on the appell matrix functions," Quaestiones Mathematicae, vol. 43, no. 3, pp. 321-334, 2019. 